\title{
Reporting of harms outcomes: a comparison of journal publications with unpublished clinical study reports of orlistat trials
}

\author{
Alex Hodkinson ${ }^{1 *}$, Carrol Gamble ${ }^{2}$ and Catrin Tudur Smith ${ }^{2}$
}

\begin{abstract}
Background: The quality of harms reporting in journal publications is often poor, which can impede the risk-benefit interpretation of a clinical trial. Clinical study reports can provide more reliable, complete, and informative data on harms compared to the corresponding journal publication. This case study compares the quality and quantity of harms data reported in journal publications and clinical study reports of orlistat trials.

Methods: Publications related to clinical trials of orlistat were identified through comprehensive literature searches. A request was made to Roche (Genentech; South San Francisco, CA, USA) for clinical study reports related to the orlistat trials identified in our search. We compared adverse events, serious adverse events, and the reporting of 15 harms criteria in both document types and compared meta-analytic results using data from the clinical study reports against the journal publications.

Results: Five journal publications with matching clinical study reports were available for five independent clinical trials. Journal publications did not always report the complete list of identified adverse events and serious adverse events. We found some differences in the magnitude of the pooled risk difference between both document types with a statistically significant risk difference for three adverse events and two serious adverse events using data reported in the clinical study reports; these events were of mild intensity and unrelated to the orlistat. The CONSORT harms reporting criteria were often satisfied in the methods section of the clinical study reports $(70-90 \%$ of the methods section criteria satisfied in the clinical study reports compared to $10-50 \%$ in the journal publications), but both document types satisfied 80-100\% of the results section criteria, albeit with greater detail being provided in the clinical study reports.

Conclusions: In this case study, journal publications provided insufficient information on harms outcomes of clinical trials and did not specify that a subset of harms data were being presented. Clinical study reports often present data on harms, including serious adverse events, which are not reported or mentioned in the journal publications. Therefore, clinical study reports could support a more complete, accurate, and reliable investigation, and researchers undertaking evidence synthesis of harm outcomes should not rely only on incomplete published data that are presented in the journal publications.
\end{abstract}

Keywords: Harms, Orlistat, Clinical study report, Adverse event, Adverse effect, Randomised controlled trial, Systematic review, Evidence-based healthcare, Obesity

* Correspondence: alex.hodkinson@york.ac.uk

${ }^{1}$ Centre for Reviews and Dissemination, University of York, Heslington, York YO10 5DD, UK

Full list of author information is available at the end of the article

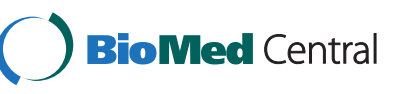

(c) 2016 Hodkinson et al. Open Access This article is distributed under the terms of the Creative Commons Attribution 4.0 International License (http://creativecommons.org/licenses/by/4.0/), which permits unrestricted use, distribution, and reproduction in any medium, provided you give appropriate credit to the original author(s) and the source, provide a link to the Creative Commons license, and indicate if changes were made. The Creative Commons Public Domain Dedication waiver (http://creativecommons.org/publicdomain/zero/1.0/) applies to the data made available in this article, unless otherwise stated. 


\section{Background}

There are two driving concerns that continue to grow when relying on published medical research to reflect the truth [1]. First, trials often remain unpublished years after completion, and the results are, therefore, unavailable to the public. Second, trials often display a distorted representation, where publications present a biased or misleading description of the design, conduct, or results of a trial $[2,3]$.

Journal publications and registry reports currently represent the main information source for obtaining summaries of clinical trial data for the purposes of clinical and health policy decision-making [4]. Results in the past have found reporting in journal publications to be inadequate and inconsistent [5], and although clinical trial registries have been responsible for making major strides in improving the transparency of trial data, a recent study suggested that the results from trial registries often remain unavailable [6].

The clinical study report (CSR) is a structured document that summarises the analysis methods and results of a clinical trial submitted for marketing authorization of an investigational medicinal product in the European Union, Japan, or the United States. CSRs are an 'integrated' full report, which can be up to a thousand pages in length, and include extensive detailed information on the efficacy and harms of interventions. The information in these documents relating to harms is usually separated individually by adverse event (AE) and serious adverse event (SAE) terms in summary tables and listings.

In the past, researchers have made major efforts to gain access to CSRs, with the intention to inform regulatory decision-making [7]. The information contained in the CSRs has proved vital when evaluating both the efficacy [8] and safety [9] of clinical interventions. Evidence from journal publications has previously been questioned, and even overturned, by findings from unpublished information reported in the CSR [10]. In December 2009, Roche was the first global healthcare company to release 'Clinical Study Reports' after growing concerns over their product Tamiflu [8]. Their policy now allows researchers to access the CSRs and summary reports used for regulatory purposes since 1 January 1999. In 2010, the European Medicine Agency (EMA) [11] became the first major regulatory agency to agree to an open-access policy to confidential documents, including CSRs. However, in 2013, the EMA was forced to step backwards when the general court of the European Union (EU) ordered them to limit the access to their reports due to legal cases from two drug companies [12]. In October 2014, the EMA published their final policy on the access to documents and CSRs [13].

Orlistat (trade name: Xenical) is marketed by Roche in most countries. It is used in the treatment of obesity as a selective inhibitor of gastric and pancreatic lipase [14].
Mild, but unpleasant, gastrointestinal (GI) side effects are commonly reported with orlistat use. A recent review [15], including 16 randomized placebo-controlled trials of orlistat, estimated an increased risk of discontinuations due to AEs of $3 \%$ (95\% CI 1-4 \%) with orlistat. The most common AEs leading to withdrawal were GI (40\%); only eight (50\%) trials specified the number of AEs due to GI problems. Another study [16] of 29 trials of orlistat indicated an increase in the risk for diarrhoea, flatulence, abdominal pain, and dyspepsia in orlistattreated patients compared with placebo. No SAEs were reported in these reviews. Concern exists that there may also be an associated increased risk of serious hepatic events, as indicated in a case series study using primary care data from the Clinical Practice Research Datalink (CPRD) [17].

We aim to carry out an exploratory review consisting of two main analyses: (1) to compare the number(s) of reported harms (AEs and SAEs) and (2) the structured reporting of harms. Both analyses will be assessed between CSRs and journal publications using a case study of Roche-sponsored orlistat trials to provide a summary of the added value, if any, from the CSRs. To our knowledge, an in-depth exploration that includes a detailed meta-analysis of this type has not been published in previous CSR-related research.

\section{Methods}

We planned to identify independent trials, each of which were reported within two different trial summary reports: CSRs and publically available journal publications. The aim was to compare these document types and determine whether there were inconsistencies in the quality and quantity of reporting of harms. The CSRs were released by Roche (Genentech; South San Francisco, CA, USA).

\section{Identifying the studies}

A search was implemented by one researcher $(\mathrm{AH})$ in the Cochrane Central register (final search 6 July 2013) and Ovid MEDLINE (final search 2 July 2013) to obtain all relevant published, randomised, controlled trials comparing orlistat against a placebo for obesity treatment. The search strategies are provided in Additional file 1. Each full article was assessed independently by one reviewer $(\mathrm{AH})$ to determine eligibility. We included published and unpublished RCTs investigating the use of orlistat. No restriction was placed on the clinical area. Observational studies and those studies that did not specify orlistat as their primary intervention were excluded.

\section{Data collection and extraction}

Roche was contacted and asked to provide the corresponding CSRs for each of the publications identified. A 
Roche CSR consists of the following five modules of information:

Module I: The 'Core report' - background and rationale, objectives, materials and methods, efficacy results, safety results, discussion, conclusion and appendices Module II: 'Study documents' - protocol and amendment history, blank case report forms (CRFs), subject information sheet and consent form, glossaries of original and preferred terms, randomization list, reporting analysis plan (RAP), certificates of analysis, list of investigators and list of ethics committee members

Module III: 'Listings of demographic and efficacy data' Module IV: 'Listing of safety data'

Module V: 'Statistical report and appendices' - statistical analysis and efficacy results

For each matching document pair (CSR and journal publication), the following data were extracted:

- Content and characteristics of both document types, including whether a clear primary objective of safety was defined, a word count of the information relating to harms in both the journal publication (including any online supplementary material) and in the CSR documents of text only (word count performed using the software AnyCount version 7.0 [18]). Missing pages relating to safety due to redactions were noted in the results; we managed to obtain these on further request.

- Name of each reported AE and SAE term recorded for both placebo and orlistat, with the overall number of patients in the safety population, as defined in the respective document. The intensity grading (i.e. mild, moderate, or severe), relationship to orlistat, and definition of the SAEs were also observed where possible. SAEs were defined as any event that was fatal or life-threatening, requiring hospitalization or prolongation of hospitalization, or an overdose. The AE coding system was also detailed.

- Reporting structure of harms (CONSORT-harms [19] used as a benchmark). The CONSORT extension for reporting harm outcomes extends ten checklist items of the CONSORT (2001) checklist to help support the reporting of harms-related data from RCTs. This includes guidance on how to report harms in the title and abstract, introduction, methods (definitions, collection, and analysis), results (withdrawals, denominators, and type), and the discussion.

One researcher $(\mathrm{AH})$ extracted, and a second reviewer (CTS) checked the data extraction. Discrepancies in the rates of agreement were resolved through consensus or recourse to a third reviewer (CG), where necessary. As there were no disagreements in the data extraction for the first three trials (NM16189, M37013, and M37002), extraction for the final two trials was only carried out by one reviewer $(\mathrm{AH})$.

\section{AEs and SAEs}

For a particular trial, all harms (AEs and SAEs) reported in either the journal publication or the CSR were extracted and compared across the two document types. The clinically validated medical terminology dictionary MedDRA is commonly used during the regulatory process by all stakeholders in healthcare; it is used for coding harm outcomes. These reported outcomes were then organized into each of the five levels of the MedDRA dictionary: the system organ class, high-level group term, high-level term, preferred term, and lowest level term. Outcomes are usually reported in the journal publications and CSRs as MedDRA preferred term level events. Therefore, we compared the total number of reported MedDRA preferred terms, and if a preferred term was reported in both the CSR and journal publication, the numerical data were compared, and any discrepancies, noted.

For each MedDRA preferred term (AE and SAE), the data extracted from the CSRs were used to estimate risk differences, which were pooled across trials using fixedeffect meta-analysis. A corresponding meta-analysis was performed using the data extracted from the journal publications wherever relevant. The pooled risk difference (RD) with $95 \%$ confidence interval [20] and the $\mathrm{I}^{2}$ statistic [21] were compared between the CSR-based and the journal publication-based analyses. As the SAE data were sparse, a sensitivity analysis was undertaken to pool the relative risk (RR). We stress that these meta-analysis results are based on a subset of the eligible trials of orlistat and are presented for the purpose of methodological comparison rather than definitive clinical results.

\section{Structured reporting of harms}

Using the CONSORT-harms extension [19] as a benchmark for reporting harms data from a randomised controlled trial, documents were assessed across 15 adapted criteria (see Table 1) that focus on the methods and results. Each trial was classified as follows for each individual criteria:

BOTH - both documents report the criteria CSR - only reported criteria in the clinical study report Pub - only reported criteria in the trial publication NR - criteria not reported in either document

The total number of criteria satisfied in each CSR and journal publication for a particular trial was calculated and expressed as a percentage of 15 criteria. 
Table 1 Fifteen criteria (adapted from the CONSORT-harms extension) assessed to evaluate the completeness of reporting methods and results of harms

\begin{tabular}{|c|c|c|c|}
\hline & Criteria & Criteria description & Description of complete reporting for criteria \\
\hline \multirow[t]{10}{*}{ Methods } & 1 & List addressed adverse events with definitions & Listed AEs with definitions (with attention to the grading, when relevant) \\
\hline & 2 & Mode for collecting data & $\begin{array}{l}\text { Full description of questionnaires, interviews, or tests used to collect information } \\
\text { on the harms. Detailed information on the questions asked }\end{array}$ \\
\hline & 3 & Timing and time frame of surveillance & $\begin{array}{l}\text { Description of the time frame of surveillance for AEs, with the stopping period } \\
\text { detailed }\end{array}$ \\
\hline & 4 & Attribution methods & $\begin{array}{l}\text { Person responsible for making attribution disclosed and whether blinding was } \\
\text { used }\end{array}$ \\
\hline & 5 & Intensity of ascertainment & Specify clearly how the withdrawals are handled in the analyses \\
\hline & 6 & Harms-related monitoring & $\begin{array}{l}\text { Plans for monitoring and rules for stopping for the benefits and harms } \\
\text { separately }\end{array}$ \\
\hline & 7 & Coding of AEs & Reference to any coding system used and person responsible for the coding \\
\hline & 8 & Handling of recurrent events & Specify how recurrent events are handled: detailed as separate events or as one \\
\hline & 9 & Timing issues & Timing of events explained, if recurrent \\
\hline & 10 & $\begin{array}{l}\text { Plans to perform any statistical analyses and } \\
\text { inferences }\end{array}$ & $\begin{array}{l}\text { Described how pre-specified statistical analyses are separated from post hoc } \\
\text { analyses, and any common problems addresses }\end{array}$ \\
\hline \multirow[t]{5}{*}{ Results } & 11 & Withdrawals and discontinuations & $\begin{array}{l}\text { Reasons for discontinuations and separated by arm. Flow diagrams used to } \\
\text { display withdrawals }\end{array}$ \\
\hline & 12 & Denominators for analyses on harms & $\begin{array}{l}\text { Analyses and definitions used and clearly stated (i.e. intention to treat (ITT)), } \\
\text { and all denominators for safety population are clearly detailed }\end{array}$ \\
\hline & 13 & Specifying AE type & Results presented separately by System Organ Classification type \\
\hline & 14 & Grading or scaling used & Each AE type should offer appropriate metrics of absolute risk \\
\hline & 15 & Seriousness per arm & Reported separately for each type of event \\
\hline
\end{tabular}

When both document types reported on any particular individual criteria (i.e. BOTH), the reported information was compared and classified as follows:

CSR (+) - The CSR provides more information than the journal publication

Similar (O) - Both document types provide equal and similar information

CSR (-) - The journal publication provides more information than the CSR

\section{Results}

Thirty-one journal publications related to 31 randomised controlled trials of orlistat were identified in our search (Fig. 1). We requested access to the full CSRs from Roche corresponding to each of these trials. The CSRs could not be provided for 26 of these trials. Of the 26 trials, 17 were not Roche-sponsored, and therefore, the CSRs were not held by Roche. Nine trials pre-dated Roche's policy extension, which only allows access to trials dating back to 1 January 1999.

CSRs were obtained and matched with the corresponding journal publication for five trials (NM16189 [17], M37013 [18], M37002 [19], M37047 [20], and BM15421 [21]). Module I of the CSR was provided for all trials. Module II was not provided for one trial (BM15421), and module $\mathrm{V}$ was not provided for one trial (NM16189). We contacted Roche to provide reasons for these missing modules and for the four missing pages. Roche informed us that these sections contained confidential information and had to be removed. Modules III and IV were not provided for any of the trial CSRs because they contained individual patient data listings.

Table 2 shows the content and characteristics for each trial document pair. Safety was not the primary objective for any of the five trial journal publications but was defined as a secondary objective in three journal publications [22-24] and was not specified in two journal publications $[25,26]$. Two trials $[23,25]$ were published in the Journal of Diabetes, Obesity and Metabolism; two trials [24, 26], in the Journal of Diabetes Care; and one trial [22], in the Journal of the American Medical Association (JAMA).

The mean word count across the five trial journal publications was 7,265 (standard deviation (sd) 1,894), with an average of $10 \%$ of words (mean (sd) 757 (287)) dedicated to safety. The CSRs had a mean (sd) of 163,411 $(96,872)$ words across all trials, with approximately $3 \%$ (mean (sd) 4,663 (1,446)) related to safety. The mean difference between the CSR and journal publication was $3,906$ (95 \% CI $(1,756 ; 6,056))$ words.

\section{Comparison of reported $\mathrm{AE}$ and $\mathrm{SAE}$ event data}

MedDRA version 2.3 had been used to code AEs and SAEs in all five trials. 


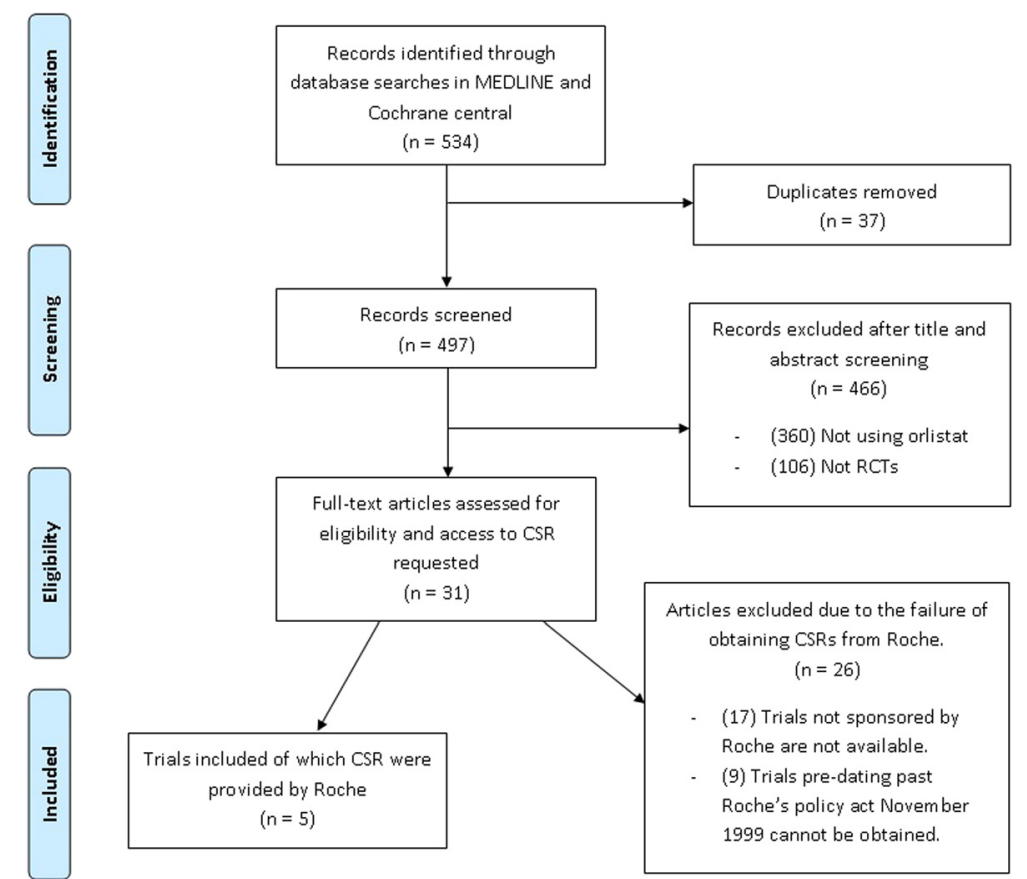

Fig. 1 Flow diagram for obtaining the trial reports

Table 2 Content and characteristics of the trial documents

\begin{tabular}{|c|c|c|c|c|c|c|c|c|c|c|}
\hline Trial ID & \multicolumn{2}{|l|}{ NM16189 } & \multicolumn{2}{|l|}{ M37013 } & \multicolumn{2}{|l|}{ M37002 } & \multicolumn{2}{|l|}{ M37047 } & \multicolumn{2}{|c|}{ BM15421 } \\
\hline Safety primary objective of trial? & \multicolumn{2}{|l|}{ Not } & \multicolumn{2}{|l|}{ Not } & \multicolumn{2}{|l|}{ No¥ } & \multicolumn{2}{|l|}{ No¥ } & \multicolumn{2}{|l|}{ Not } \\
\hline $\begin{array}{l}\text { Journal publication: author, } \\
\text { journal and year }\end{array}$ & \multicolumn{2}{|c|}{$\begin{array}{l}\text { Chanoine [22], Journal } \\
\text { of the American Medical } \\
\text { Association (JAMA (2005) }\end{array}$} & \multicolumn{2}{|c|}{$\begin{array}{l}\text { Halpern [23], Diabetes, } \\
\text { Obesity and Metabolism } \\
\text { (2003) }\end{array}$} & \multicolumn{2}{|c|}{$\begin{array}{l}\text { Hanefeld [25], Diabetes, } \\
\text { Obesity and Metabolism } \\
\text { (2002) }\end{array}$} & \multicolumn{2}{|c|}{$\begin{array}{l}\text { Kelley [26], Diabetes } \\
\text { Care (2002) }\end{array}$} & \multicolumn{2}{|c|}{$\begin{array}{l}\text { Torgerson [24], } \\
\text { Diabetes } \\
\text { Care (2004) }\end{array}$} \\
\hline $\begin{array}{l}\text { CSR research report no. } \\
\text { (date of CSR) }\end{array}$ & \multicolumn{2}{|c|}{$1011426(2003)$} & \multicolumn{2}{|c|}{$1002688(2000)$} & \multicolumn{2}{|c|}{$1003882(2001)$} & \multicolumn{2}{|c|}{$1002743(2001)$} & \multicolumn{2}{|c|}{$1008213(2002)$} \\
\hline \multicolumn{11}{|c|}{ Word count (including text and numbers, but not tables) } \\
\hline Trial document & Pub & CSR & Pub & CSR & Pub & CSR & Pub & CSR & Pub & CSR \\
\hline $\begin{array}{l}\text { Total number of words } \\
\text { in document }\end{array}$ & 10,568 & 146,801 & 6,371 & 45,464 & 6,382 & 140,166 & 7,090 & 170,347 & 5915 & 314,277 \\
\hline $\begin{array}{l}\text { Total number of words relating } \\
\text { to safety (\% of total) }\end{array}$ & $1,147(10.9)$ & $4,883(3.3)$ & 908 (14.3) & $2,664(5.9)$ & $638(10)$ & $4,964(3.5)$ & 707 (10) & $4,150(2.4)$ & $\begin{array}{l}387 \\
(6.5)\end{array}$ & $\begin{array}{l}6,653 \\
(2.1)\end{array}$ \\
\hline \multicolumn{11}{|l|}{$\begin{array}{l}\text { CSR Module }{ }^{\phi} \text { supplied by } \\
\text { Roche }\end{array}$} \\
\hline । & \multicolumn{2}{|l|}{$\boldsymbol{J}^{\Pi}$} & \multicolumn{2}{|l|}{$\boldsymbol{v}^{\Pi}$} & \multicolumn{2}{|l|}{$\checkmark$} & \multicolumn{2}{|l|}{$\boldsymbol{v}^{\Pi}$} & \multicolumn{2}{|l|}{$\boldsymbol{J}^{\Pi}$} \\
\hline$\|$ & \multicolumn{2}{|l|}{$\checkmark$} & \multicolumn{2}{|l|}{$\checkmark$} & \multicolumn{2}{|l|}{$\checkmark$} & \multicolumn{2}{|l|}{$\checkmark$} & \multicolumn{2}{|l|}{ * } \\
\hline III & \multicolumn{2}{|l|}{$*$} & \multicolumn{2}{|l|}{$*$} & \multicolumn{2}{|l|}{ * } & \multicolumn{2}{|l|}{ * } & \multicolumn{2}{|l|}{ * } \\
\hline IV & * & & $*$ & & * & & * & & * & \\
\hline V & * & & $\checkmark$ & & $\checkmark$ & & $\checkmark$ & & $\checkmark$ & \\
\hline
\end{tabular}

Footnote:

CSR Clinical study report, Pub Journal publication

†Safety secondary objective in both the CSR and journal publication; ¥Objective to assess improvements in glycaemic control and cardiovascular disease risk in both CSR and Journal publication; ${ }^{\phi}$ Module: I = Core report (background and rationale, objectives, materials and methods, efficacy results, safety results, discussion, conclusion and appendices); II = Study documents (protocol and amendments history, black case report form (CRF), subject information sheet and consent form, glossaries of original and preferred terms, randomization list, reporting analysis plan (RAP), certificates of analysis, list of investigators, list of ethics committee members); III = Listing of demographic and efficacy data; IV = Listing of safety data; $\mathbf{V}=$ Statistical reports and appendices (Statistical analysis, efficacy results). $\checkmark$ Module provided in CSR; *Roche did not provide these modules, since they contained individual patient data listings and therefore were deleted. $\epsilon$ We could only count words for modules that were made available by Roche, so the actual number would be greater than this. The percentage of words relating to harms would therefore differ; ${ }^{\Pi}$ CSRs each had one missing page in module I, which Roche provided upon further requests. Any additional information from this was used in the results. 


\section{Adverse events}

The total number of MedDRA preferred terms for AEs varied across trials (Fig. 2) (Forest plots are provided in Additional file 1). The journal publications did not always report the complete list of terms identified in the corresponding CSR, but all of these 'missing' AEs were of mild to moderate intensity and were unrelated to the intervention. For instance, in one trial (M37013), very good consistency in reporting was observed between the CSR and journal publication, with 18 AEs reported in total, 18 (100\%) of which were listed in the CSR and 17 (94 \%) in the journal publication. However, very poor consistency was observed for the three trials (M37002, M37047, and BM15421), with $5 \%$ or fewer of the total AEs being reported in the journal publication (M37002, one (5 \%); M37047, one (4\%); BM15421, 0 (0 \%)). When a MedDRA preferred term was listed in both the CSR and journal publication, complete agreement was observed in the numerical results (Additional file 2) except for one trial (M37013), where three additional patients with 'abdominal pain' on orlistat were identified within the journal publication.

In the meta-analysis (MA) for the AEs (Table 3), 61 individual MedDRA preferred terms were reported in either the CSR or journal publication across the five trials (Additional file 1). Thirty (49\%) of these terms were reported in the CSR and corresponding journal publication for at least one trial, thereby allowing a comparison of the pooled results. In six $(20 \%)$ of the 30 MA comparisons, the magnitude of the effect differed (the $95 \%$ CI for the pooled risk difference (RD) did not overlap between the CSR and the journal publication results). These include the AE terms: 'increased defecation,' 'oily spotting,' 'oily evacuation, 'faecal incontinence, 'soft stools', and 'faecal urgency'. For the $31 \mathrm{AE}$ terms that had only been reported in a CSR, $23(74 \%)$ analyses suggested an increased risk of an $\mathrm{AE}$ on orlistat, two (6\%) of which were statistically significant (faeces discolouration and dry skin); these AEs were mild and were unrelated to treatment. For four (13\%) terms, an increased risk of an event occurred with the placebo, one (3\%) of which was statistically significant (haemorrhoids) and of a mild grade.

\section{Serious adverse events}

The total number of MedDRA preferred terms for SAEs were generally poorly reported in journal publications (Fig. 3; Additional file 3). For the four trials (M37013, M37002, M37047, and BM15421) only $11 \%$ or fewer of the total SAE terms were reported in the journal publication with $11 \%, 0 \%, 0 \%$, and $0 \%$, respectively. All SAEs that were reported only in the CSR were of mild intensity grading and were unrelated to the treatment.

In trial NM16189, 19 SAE terms were reported across the CSR and journal publication. Thirteen of these were reported in both documents, either with full numerical agreement (12 SAE terms) or with disagreement in numerical results (one depression SAE on orlistat reported in the CSR, and two depression SAEs reported in the journal publication) (Additional file 3). Five SAE terms were only reported in the CSR (demyelination (one) and bronchospasm aggravated (one) on placebo, and convulsions (one), suicidal ideation (one) and liquid stools (one) on orlistat). Encephalomyelitis as an SAE was reported for placebo in the publication but not the CSR. Trial M37013 reports nine SAEs, with only "diarrhoea and dehydration" on orlistat reported in both documents. The remaining eight

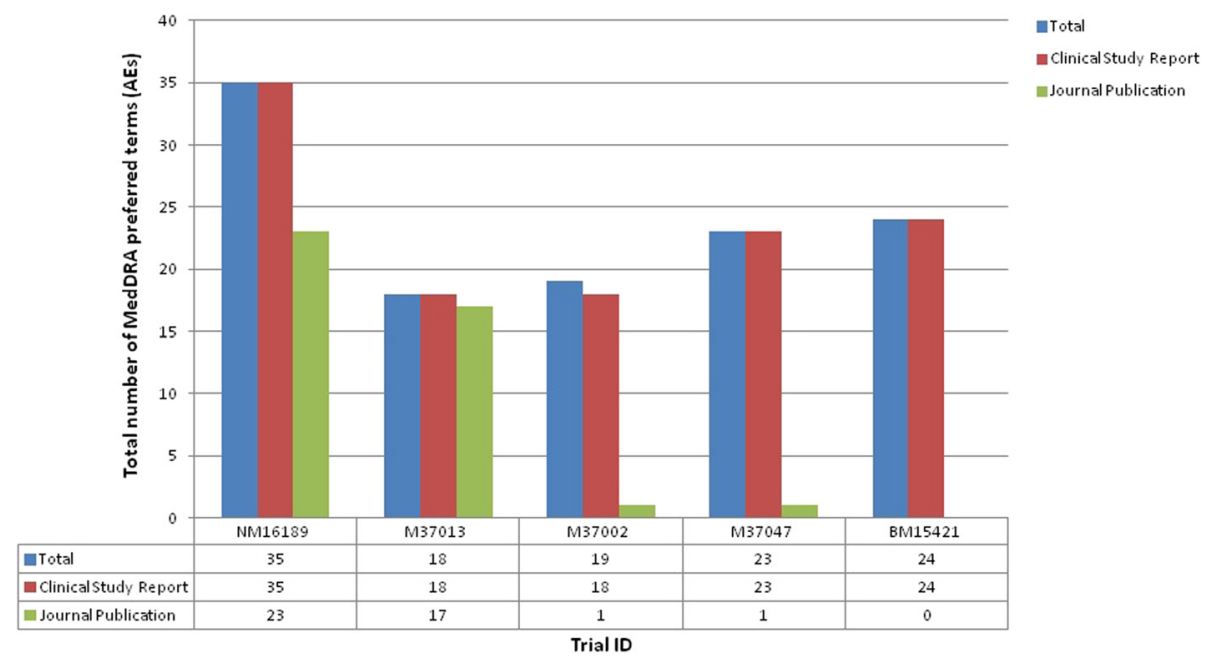

Fig. 2 The total number of MedDRA preferred terms (Adverse Events) reported in clinical study reports (CSRs) and journal publications across all five trials. Footnote: Total: Total number of individual MedDRA preferred terms related to AEs reported across the CSR and journal publication for a trial 
Table 3 Summary of meta-analysis results for the individual MedDRA preferred term adverse events pooled across all five trials

\begin{tabular}{|c|c|c|c|c|}
\hline \multirow{2}{*}{$\begin{array}{l}\text { Adverse events (AEs) } \\
\text { Meta-analysis characteristic }\end{array}$} & \multirow[b]{2}{*}{ Total } & \multicolumn{3}{|l|}{ Breakdown of adverse events reporting } \\
\hline & & $\begin{array}{l}\text { Once in the clinical study report (CSR) } \\
\text { and journal publication }\end{array}$ & CSR & Journal publication \\
\hline $\begin{array}{l}\text { Number of AE terms reported } \\
\text { (\% of total) }\end{array}$ & 61 & $30(49 \%)$ & $31(51 \%)$ & $0(0)$ \\
\hline $\begin{array}{l}\text { Direction of pooled risk effect } \\
\text { in meta-analysis }\end{array}$ & & $\begin{array}{l}\text { For all } 30 \text { AEs there is agreement in } \\
\text { direction of the pooled risk effect } \\
\text { between the pairing of documents }\end{array}$ & $\begin{array}{l}\text { - } 23(74 \%) \text { showed an increased pooled risk } \\
\text { of AE on orlistat } \\
\text { - four }(13 \%) \text { showed no difference } \\
\text { - four }(13 \%) \text { showed increased pooled risk } \\
\text { of AE on placebo }\end{array}$ & \\
\hline $\begin{array}{l}\text { AE listings for when there is } \\
\text { a change in effect including } \\
\text { statistical significance }\end{array}$ & & $\begin{array}{l}\text { - Pooled risk effect was greater in } \\
\text { journal publication for four AEs; } \\
\text { increased defecation, oily spotting, } \\
\text { oily evacuation, faecal incontinence } \\
\text { - Pooled risk effect was greater in } \\
\text { the CSR for two AEs; soft stools, } \\
\text { faecal urgency }\end{array}$ & $\begin{array}{l}\text { - two (6\%) of the } 23 \text { AEs were statistically } \\
\text { significant; faeces discolouration, dry skin } \\
\text { - one (3\%) of the four AEs with } \\
\text { increased risk on placebo was statistically } \\
\text { significant; haemorrhoids }\end{array}$ & \\
\hline
\end{tabular}

Footnote:

${ }^{\mathrm{a}}$ These adverse events were mild and unrelated to treatment

SAEs were only reported in the CSR; death (one), diabetes mellitus (one), hysterectomy and perineoplasty (one), mitral lesion (one) on placebo and Chronic cholecystitis (one), nephrectomy due to previous renal carcinoma (one), nephrectomy and lithotripsy due to previous nephrolithiasis (one), ovary carcinoma and ascites (one) on orlistat. The three remaining trials (M37002, M37047, and BM15421) report a high number of SAEs (40, 53, and 255) within the CSR that have not been reported in the corresponding journal publication.

In the MA for the SAEs (Table 4), 326 individual terms were reported in either the CSR or journal publication across the five trials (Additional file 4). Fourteen (4\%) of these terms were reported in the CSR and corresponding journal publication for at least one trial, allowing a comparison of the pooled results. For the 311 (95\%) terms that had only been reported in a CSR, 16 (5\%) analyses suggested an increased risk of an SAE on orlistat, two (13\%) of which were statistically significant (carotid artery stenosis and varicose veins), but all were mild and unrelated. In the sensitivity analysis, pooling relative risk rather than risk differences, no SAEs were found to be statistically significant. However, we were unable to estimate the pooled relative risk for ten AEs (including carotid artery stenosis and varicose veins), as they include multiple studies reporting no events in the placebo group.

\section{Structured reporting of harms}

The quality of reporting harms-related information, as assessed against the 15 criteria adapted from the CONSORT-harms checklist, are displayed in Table 5.

The CSRs satisfied 70-90 \% the methods related criteria across the five trials compared to the journal

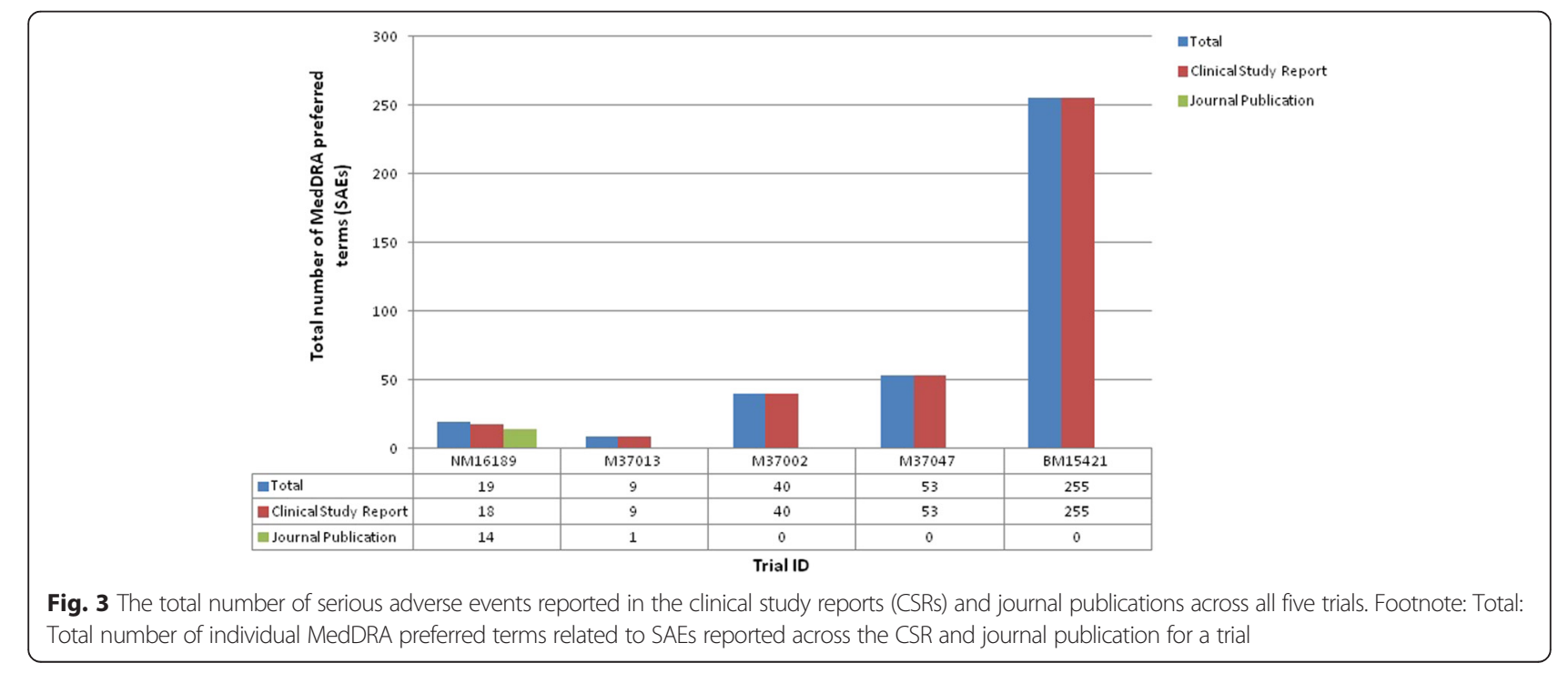


Table 4 Summary of meta-analysis results for the individual MedDRA preferred term serious adverse events pooled across all five trials

\begin{tabular}{|c|c|c|c|c|}
\hline \multirow{2}{*}{$\begin{array}{l}\text { Serious Adverse Events (SAEs) } \\
\text { Meta-analysis characteristic }\end{array}$} & \multirow[b]{2}{*}{ Total } & \multicolumn{3}{|c|}{ Breakdown of serious adverse events reporting } \\
\hline & & $\begin{array}{l}\text { Once in the clinical study report (CSR) } \\
\text { and journal publication }\end{array}$ & CSR & Journal publication \\
\hline $\begin{array}{l}\text { Number of SAE terms } \\
\text { reported (\% of total) }\end{array}$ & 326 & $14(4 \%)$ & $311(95 \%)$ & $1(<1 \%)$ \\
\hline $\begin{array}{l}\text { Direction of the pooled risk } \\
\text { effect in the meta-analysis }\end{array}$ & & $\begin{array}{l}\text { For all } 14 \text { SAEs, there is agreement } \\
\text { in direction of the pooled risk effect } \\
\text { between the pairing of documents }\end{array}$ & $\begin{array}{l}\text { - } 16(5 \%) \text { showed increased pooled risk of } \\
\text { SAE on orlistat } \\
\text { - } 281(90 \%) \text { showed no difference } \\
\text { - } 14(5 \%) \text { showed an increased pooled risk } \\
\text { of SAE on placebo }\end{array}$ & $\begin{array}{l}\text { The one SAE showed } \\
\text { increased pooled risk } \\
\text { on placebo }\end{array}$ \\
\hline $\begin{array}{l}\text { SAE listings for when there } \\
\text { is a change in effect including } \\
\text { statistical significance }\end{array}$ & & & $\begin{array}{l}\text { Two }(13 \%) \text { of the } 16 \text { SAEs were } \\
\text { statistically significant; carotid artery } \\
\text { stenosis, varicose veins }{ }^{\mathrm{a}}\end{array}$ & $\begin{array}{l}\text { One SAE; encephalomyelitis } \\
\text { was statistically } \\
\text { non-significant }\end{array}$ \\
\hline
\end{tabular}

Footnote:

${ }^{a}$ These serious adverse events were mild and unrelated to treatment

publications, which satisfied between $10 \%$ and $50 \% .3$ timing and time frame of surveillance for AEs). Both The CSRs consistently provided much greater detail re- the CSRs and the journal publications satisfied 80-100 garding planned analyses than the journal publication, \% of criteria in their results sections, but greater detail and on only one occasion did the journal publication was generally provided in the CSR. This included full provide greater detail than the CSR (trial M37013; item summary tables of the AE and SAE data, including

Table 5 Comparison of 15 harms criteria (CONSORT-harms extension used as a benchmark)

\begin{tabular}{|c|c|c|c|c|c|c|c|}
\hline & \multirow[b]{2}{*}{ Criteria } & \multirow[b]{2}{*}{ Description of item } & \multicolumn{5}{|l|}{ Trial ID } \\
\hline & & & NM16189 & M37013 & M37002 & M37047 & BM15421 \\
\hline \multirow[t]{12}{*}{ Methods criteria } & 1 & List addressed adverse events (AEs) with definitions. & CSR & CSR & CSR & CSR & CSR \\
\hline & 2 & Mode of collecting harms data. & BOTH ${ }^{\mathrm{b}}$ & $\mathrm{BOTH}^{\mathrm{b}}$ & $\mathrm{BOTH}{ }^{\mathrm{b}}$ & CSR & $\mathrm{BOTH}^{\mathrm{a}}$ \\
\hline & 3 & Timing and time frame of surveillance for adverse events. & BOTH ${ }^{b}$ & Pub & CSR & NR & $\mathrm{BOTH}^{\mathrm{a}}$ \\
\hline & 4 & Attribution methods. & CSR & NR & CSR & NR & NR \\
\hline & 5 & Intensity of ascertainment. & CSR & $\mathrm{BOTH}{ }^{\mathrm{b}}$ & CSR & CSR & CSR \\
\hline & 6 & Harms related monitoring. & CSR & BOTH ${ }^{b}$ & CSR & CSR & CSR \\
\hline & 7 & Coding of AEs. & CSR & CSR & $\mathrm{BOTH}^{\mathrm{a}}$ & CSR & CSR \\
\hline & 8 & Handling of recurrent events. & NR & CSR & NR & CSR & NR \\
\hline & 9 & Timing issues. & CSR & CSR & CSR & NR & CSR \\
\hline & 10 & Plans to perform any statistical analyses and inferences. & CSR & $\mathrm{BOTH}^{\mathrm{a}}$ & $\mathrm{BOTH}{ }^{\mathrm{a}}$ & $\mathrm{BOTH}{ }^{\mathrm{a}}$ & $\mathrm{BOTH}^{\mathrm{a}}$ \\
\hline & \multicolumn{2}{|c|}{$\begin{array}{l}\text { Total items satisfied for methods criteria in clinical study report (CSR) (\% of total } \\
10 \text { items assessed) }\end{array}$} & $9(90)$ & $8(80)$ & $9(90)$ & $7(70)$ & $8(80)$ \\
\hline & \multicolumn{2}{|c|}{$\begin{array}{l}\text { Total items satisfied for methods criteria in publication (\% of total ten items } \\
\text { assessed) }\end{array}$} & $2(20)$ & $5(50)$ & $3(30)$ & $1(10)$ & $3(30)$ \\
\hline \multirow[t]{7}{*}{ Results criteria } & 11 & Withdrawals and discontinuations. & $\mathrm{BOTH}^{\mathrm{a}}$ & $\mathrm{BOTH}^{\mathrm{a}}$ & BOTH ${ }^{\text {a }}$ & $\mathrm{BOTH}^{\mathrm{a}}$ & CSR \\
\hline & 12 & Denominators for analyses on harms. & ВОTH ${ }^{b}$ & $\mathrm{BOTH}^{\mathrm{b}}$ & BOTH ${ }^{\text {a }}$ & CSR & $\mathrm{BOTH}^{\mathrm{b}}$ \\
\hline & 13 & Specifying AE type. & ВOTH ${ }^{a}$ & $\mathrm{BOTH}^{\mathrm{a}}$ & $\mathrm{BOTH}^{\mathrm{a}}$ & $\mathrm{BOTH}{ }^{\mathrm{a}}$ & $\mathrm{BOTH}{ }^{\mathrm{a}}$ \\
\hline & 14 & Grading or scaling used. & NR & $\mathrm{BOTH}^{\mathrm{a}}$ & $\mathrm{BOTH}^{\mathrm{a}}$ & $\mathrm{BOTH}{ }^{\mathrm{a}}$ & $\mathrm{BOTH}^{\mathrm{a}}$ \\
\hline & 15 & Seriousness per arm. & ВOTH ${ }^{\text {a }}$ & $\mathrm{BOTH}^{\mathrm{a}}$ & $\mathrm{BOTH}^{\mathrm{a}}$ & $\mathrm{BOTH}{ }^{\mathrm{a}}$ & $\mathrm{BOTH}^{\mathrm{a}}$ \\
\hline & \multicolumn{2}{|c|}{ Total items satisfied for results criteria in the CSR (\% of total five items assessed) } & $4(80)$ & $5(100)$ & $5(100)$ & $5(100)$ & $5(100)$ \\
\hline & \multicolumn{2}{|c|}{$\begin{array}{l}\text { Total items satisfied for results criteria in the publication (\% of total five items } \\
\text { assessed) }\end{array}$} & $4(80)$ & $5(100)$ & $5(100)$ & $4(80)$ & $4(80)$ \\
\hline \multicolumn{3}{|c|}{ Total items satisfied in CSR (\% of total 15 items assessed) } & $13(87)$ & $13(87)$ & $14(93)$ & $12(80)$ & $13(87)$ \\
\hline \multicolumn{3}{|c|}{ Total items satisfied in publication (\% of total 15 items assessed) } & $6(40)$ & $10(67)$ & $8(53)$ & $5(33)$ & $7(47)$ \\
\hline
\end{tabular}

\section{Footnote:}

BOTH 'reported in CSR and the corresponding journal publication', CSR 'only reported within the CSR', Pub 'only reported in journal publication', NR 'neither

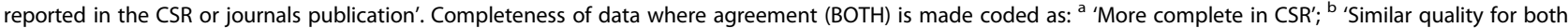
documents'; - 'less complete in the CSR' 
withdrawals due to harm, severity grading, and denominators for the numbers included in the safety population.

\section{Discussion}

This case study has shown differences in the completeness and quality of reporting harms-related information between journal publications and CSRs for five orlistat trials. Information on the patient-relevant harm outcomes, including SAEs, which is required for unbiased trial evaluation, was missing from the publicly available journal article. Including these missing data from the CSRs altered the magnitude of the pooled risk difference estimates in a few cases and even resulted in five statistically significant differences (including three AEs and two SAEs). The statistically significant risk differences for AEs were faeces discolouration, dry skin, and haemorrhoids, and for SAEs, carotid artery stenosis and varicose veins. However, the statistical significance of these SAEs could not be confirmed in a sensitivity analysis pooling relative risks $[27,28]$ due to zero events. The events were graded mild and were classified as unrelated to treatment. Overall, the results from the journal publications in this study follow findings from past studies $[15,16]$, with a more detailed meta-analysis showing predominantly mild gastrointestinal harm outcomes.

The quality of reporting between journal publications and CSRs showed inconsistencies when assessed by the CONSORT-harms reporting criteria. At 70-90 \%, the methods section criteria were more often satisfied in the CSRs, compared to only $10-50 \%$ of the criteria in the journal publications. However, both document types satisfied $80-100 \%$ of the results section criteria, albeit with greater detail being provided in the CSR. The journal publication was often incomplete when reporting planned analyses and summary tables of AEs and SAEs, which were missing information on withdrawals, severity grading, and numbers in the safety population. Journal publications are often impeded by word count restrictions. However, inadequate reporting of harms is still noticeable, even after the release of the CONSORT-harms extension [19], as the findings from our recent review [29] suggest. In contrast, CSRs have no such word restrictions imposed, and theoretically, all relevant information should be included. An alternative and more viable solution appears to be that journals should require more thorough reporting of harms via online supplements (e.g. de-identified CSRs, study protocols, and complete tables of AE-related information) [30].

In a recent study [4], findings on harms information obtained from the CSRs were found to be more complete and robust compared with the corresponding publically available sources (journal publications and registry reports). More than $86 \%$ of all harm outcomes (AEs and SAEs) were available from the CSRs, compared to only $26 \%$ from the journal publications. Combining harms data from registry reports and journal publications increased the proportion of outcomes to $43 \%$. Furthermore, withdrawals due to AEs were detailed completely in $91 \%$ of the CSRs, with only $51 \%$ of the journal publications providing complete information. In another study [31], inadequate reporting of the harms was shown in the Medtronic manufactured product, recombinant human bone morphogenetic protein 2 (rhBMP-2), used in spinal fusion surgery. As in our investigation, harms data were found to be missing from the publications, with considerably more data found in the corresponding trial CSRs. Further evidence of poor reporting of benefits and harms was found in a recent investigation of the product duloxetine in patients with major depressive disorder [32]. The CSRs contained extensive data on major harms that were unavailable in the journal publications and in trial registry reports. Restricting evidence synthesis to journal publications would effectively miss these important harms. Further empirical comparisons such as ours, in different clinical areas, would be valuable.

The drive to make clinical trial data more accessible has garnered widespread international support, with funders, academics, pharmaceutical industry, publishers and regulators supporting the move towards greater transparency. For example, the BMJ recently stated that it will no longer publish trials of drugs or devices where the authors do not commit to making the relevant anonymised patient-level data available; this was to be extended to all submitted clinical trials beginning 1 July 2015. In addition, the EMA has now adopted their new policy, making clinical trials data more accessible [13], including access to full CSRs. Roche should also be commended for voluntarily submitting their data and allowing further access to their CSRs. The new EU clinical trial regulation [33] published on 27 May 2014 also states under section (67) that trial data should be publically accessible and presented in an easily searchable format, with related data and documents (including trial protocol and CSR) linked together by the EU trial number'.

Our study has a number of limitations. First of all, the meta-analysis results do not provide comprehensive unbiased clinical results, as they are based only on a subset of the five eligible orlistat trials, due to the inability to obtain CSRs for the remaining 26 identified trials, which were not Roche sponsored or pre-dated Roche's policy (dating back to 1 January 1999). The meta-analyses were conducted without any adjustment for multiplicity, meaning that there is an increased chance of a false positive result, and the results should be interpreted with caution. In addition, for the five CSRs obtained from Roche in this study, some of the reports failed to include any information from modules II, III, IV, and V, and some had missing pages. Individual participant-level data and potentially 
other important information on harms are often presented in Roche's CSR modules III-V. Access to these modules and confidential patient listings may have been restricted due to privacy violations, and these missing sections could present a possible cause of bias in the results. In a recent study [34], reviewers re-analysed one of SmithKline Beecham's studies by requesting and accessing the full individual participant level data sets to compare the efficacy and safety of paroxetine. The findings from this study support the necessity of making trial individual participant-level data and protocols available to help evidence-based decisions. In module I of the CSRs, they also detailed that only commonly observed AEs (defined as those events with incidence rate in orlistat group of $\geq 5 \%$ ) were summarized, indicating that there are potentially more unreported AEs missing from the primary trial data. Therefore, the results in this study were based only on the information available.

\section{Conclusions}

This case study confirms that CSRs can provide more complete and robust information on harms data collected in clinical trials, compared to publically available journal publications. CSRs often provide extensive information about the study methods, including design, conduct, and analysis of the trial. On the other hand, these reports are able to supplement journal publications to help facilitate the assessment of risk of bias in evidence synthesis of harm outcomes. Consequently, restricting an evidence synthesis to journal publications could have implications to systematic reviewers and other stakeholders involved in healthcare research when reaching reliable conclusions about the harmful effects of medical interventions.

\section{Additional files}

Additional file 1: Forest plots for AEs and Search Criteria.pdf', Figure S1: Forest plots for all adverse event MedDRA preferred terms reported in CSR and journal publications and Table S1: Search criteria used in both Cochrane central and MEDLINE. (PDF $2276 \mathrm{~kb}$ )

Additional file 2: Reporting of AEs.xls', Table S2 Reporting of adverse events in Clinical Study Reports (CSRs) and journal articles for Olistat trials. (XLS $31 \mathrm{~kb}$ )

Additional file 3: Reporting of SAEs.xls', Table S3 Reporting of serious adverse events (SAEs) in Clinical study reports (CSRs) and journal articles for Olistat trials. (XLS $65 \mathrm{~kb}$ )

Additional file 4: SAEs Meta-Analysis results.xIs', Table S4 SAEs MetaAnalysis results. (XLS $76 \mathrm{~kb})$

\section{Abbreviations}

AEs: adverse events; BMJ: British Medical Journal; CONSORT: Consolidated Standards of Reporting Trials; CPRD: Clinical Practice Research Data-link; CSRs: clinical study reports; EMA: European Medicines Agency; EU: European Union; JAMA: Journal of the American Medical Association; MA: meta-analysis; MedDRA: Medical Dictionary for Regulatory Activities; RAP: reporting analysis plan; RD: risk difference; RR: relative risk; SAEs: serious adverse events; Sd: standard deviation.

\section{Competing interests}

All authors have completed the ICMJE uniform disclosure form and declare that there was no support from any organisation for submitted work, and that there were no financial conflicts of interest.

This work was supported by the award of a Capacity-Building Studentship to AH from the Medical Research Council (MRC) (grant number G1000397 - 1/1) North West Hub for Trials Methodology Research, UK (grant number G0800792). Since CSRs were redacted and prepared by Roche (Genetach; South San Francisco, CA, USA), patient consent was already obtained from the published trials.

The data-extraction form and protocol are available on request from ahoddy@liverpool.ac.uk

\section{Authors' contributions}

$\mathrm{AH}$ carried out all screening of the literature. AH extracted the data, and CTS and CG checked for consistency. AH, CTS, and CG interpreted the results and drafted the manuscript. All authors read and approved the final manuscript.

\section{Acknowledgements}

We are grateful to Roche for redacting and preparing the clinical study reports for use in this research, and for responding to queries relating to the documents. All analyses in this study were explained clearly within the protocol, which was also included as part of the initial data request to Roche. Apart from providing the CSRs, Roche did not have any involvement in the study. No funding was provided for any of this work.

\section{Author details}

${ }^{1}$ Centre for Reviews and Dissemination, University of York, Heslington, York YO10 5DD, UK. ${ }^{2}$ Department of Biostatistics, MRC North West Hub for Trials Methodology Research, University of Liverpool, Liverpool, England, UK.

Received: 19 August 2015 Accepted: 17 March 2016

Published online: 22 April 2016

\section{References}

1. McGauran N, Wieseler B, Kreis J, Schüler YB, Kölsch H, Kaiser T. Reporting bias in medical research - a narrative review. Trials. 2010;11:37. doi:10.1186/ 1745-6215-11-37.

2. Vedula SS, Tianjing L, Dickersin K. Differences in reporting of analyses in internal company documents versus published trial reports: comparisons in industry-sponsored trials in off-label uses of gabapentin. PLoS Med. 2013; 10(1):1-13.

3. Song F, Parekh S, Hooper L, Loke YK, Ryder J, Sutton AJ, et al. Dissemination and publication of research findings: an updated review of related biases. Health Technol Assess. 2010;14(8):iii. doi:10.3310/hta14080. ix-xi, 1-193.

4. Wieseler B, Wolfram N, McGauran N, Kerekes MF, Vervölgyi V, Kohlepp P. Completeness of reporting of patient-relevant clinical trial outcomes: comparison of unpublished clinical study reports with publicly available data. PLoS Med. 2013;10(10):e1001526. doi:10.1371/journal.pmed.1001526.

5. Moher D, Jones A, Lepage L. CONSORT Group. Use of the CONSORT statement and quality of reports of randomized trials: a comparative beforeand-after evaluation. JAMA. 2001;285(15):1992-5.

6. Prayle AP, Hurley MN, Smyth AR. Compliance with mandatory reporting of clinical trial results on ClinicalTrials.gov: cross sectional study. BMJ. 2012;344: d7373. doi:10.1136/bmj.d7373.

7. Doshi $\mathrm{P}$, Jefferson $\mathrm{T}$, del Mar C. The imperative to share clinical study reports: recommendations from the Tamiflu experience. PLoS Med. 2012;9(4):e1001201. doi:10.1371/journal.pmed.1001201.

8. Jefferson T, Jones M, Doshi P, Del Mar C. Possible harms of oseltamivir-a call for urgent action. Lancet. 2009;374(9698):1312-3.

9. Jureidini JN, McHenry LB, Mansfield PR. Clinical trials and drug promotion: selective reporting of study 329. Int J Risk Saf Med. 2008;20(1):73-81.

10. Eyding D, Lelgemann M, Grouven U, Härter M, Kromp M, Kaiser T. Reboxetine for acute treatment of major depression: systematic review and meta-analysis of published and unpublished placebo and selective serotonin reuptake inhibitor controlled trials. BMJ. 2010;341:c4737. doi:10. 1136/bmj.c4737.

11. Gøtzsche PC, Jørgensen AW. Opening up data at the European Medicines Agency. BMJ. 2011;342:d2686. doi:10.1136/bmj.d2686. 
12. Dyer $C$. European drug agency's attempts to improve transparency stalled by legal action from two US drug companies. BMJ. 2013;346:f3588. doi:10. 1136/bmj.f3588.

13. European Medicines Agency (EMA) policy on publication of clinical data for medicinal products for human use. 2nd October 2014 EMA/240810/2013. Policy/0070. Available at: http://www.ema.europa.eu/docs/en GB/ document_library/Other/2014/10/WC500174796.pdf. Accessed 23 Apr 2015.

14. Guerciolini R. Mode of action of orlistat. Int J Obes (Lond). 1997;21 Suppl 3:S12-23.

15. Johansson K, Neovius K, DeSantis SM, Rössner S, Neovius M. Discontinuation due to adverse events in randomized trials of orlistat, sibutramine and rimonabant: a meta-analysis. Obes Rev. 2009;10(5):564-75.

16. Li Z, Maglione M, Tu W, Mojica W, Arterburn D, Shugarman LR, et al. Metaanalysis: pharmacologic treatment of obesity. Ann Intern Med. 2005;142(7): 532-46.

17. Douglas IJ, Langham J, Bhaskaran K, Brauer R, Smeeth L. Orlistat and the risk of acute liver injury: self controlled case series study in UK Clinical Practice Research Datalink. BMJ. 2013;346:f1936.

18. Any Count Software. Software for word counting for PDF file. Available at: http://www.anycount.com/. Accessed 18 Nov 2014.

19. loannidis JP, Evans SJ, Gøtzsche PC, O'Neill RT, Altman DG, Schulz K, et al. Better reporting of harms in randomized trials: an extension of the CONSORT statement. Ann Intern Med. 2004;141(10):781-8.

20. Inside-R. A community Site for $R$ - sponsored by Revolution Analytics. $R$ packages: metabin meta-analysis of binary outcome data. Last Accessed 16 June 2015. Available at: http://www.inside-r.org/packages/cran/meta/docs/ metabin.

21. Higgins JPT, Thompson SG, Deeks JJ, Altman DG. Measuring inconsistency in meta-analyses. BMJ. 2003;327(7414):557-60.

22. Chanoine JP, Hampl S, Jensen C, Boldrin M, Hauptman J. Effect of orlistat on weight and body composition in obese adolescents: a randomized controlled trial. JAMA. 2005;293(23):2873-83. Erratum in: JAMA. 2005;294(12):1491.

23. Halpern A, Mancini MC, Suplicy H, Zanella MT, Repetto G, Gross J, et al. LatinAmerican trial of orlistat for weight loss and improvement in glycaemic profile in obese diabetic patients. Diabetes Obes Metab. 2003;5(3):180-8.

24. Torgerson JS, Hauptman J, Boldrin MN, Sjöström L. XENical in the Prevention of Diabetes in Obese Subjects (XENDOS) study: a randomized study of orlistat as an adjunct to lifestyle changes for the prevention of type 2 diabetes in obese patients. Diabetes Care. 2004;27(1):155-61.

25. Hanefeld M, Sachse G. The effects of orlistat on body weight and glycaemic control in overweight patients with type 2 diabetes: a randomized, placebocontrolled trial. Diabetes Obes Metab. 2002;4(6):415-23.

26. Kelley DE, Bray GA, Pi-Sunyer FX, Klein S, Hill J, Miles J, Hollander P. Clinical efficacy of orlistat therapy in overweight and obese patients with insulintreated type 2 diabetes: a 1-year randomized controlled trial. Diabetes Care. 2002;25(6):1033-41.

27. Bradburn MJ, Deeks JJ, Berlin JA, Russell LA. Much ado about nothing: a comparison of the performance of meta-analytical methods with rare events. Stat Med. 2007;26(1):53-77.

28. Sutton AJ, Cooper NJ, Lambert PC, Jones DR, Abrams KR, Sweeting MJ. Meta-analysis of rare and adverse event data. Expert Rev Pharmacoecon Outcomes Res. 2002;2(4):367-79.

29. Hodkinson A, Kirkham JJ, Tudur-Smith C, Gamble C. Reporting of harms data in RCTs: a systematic review of empirical assessments against the CONSORT harms extension. BMJ Open. 2013;3(9):e003436. doi:10.1136/ bmjopen-2013-003436.

30. Doshi P, Dickersin K, Healy D, Vedula SS, Jefferson T, et al. Restoring invisible and abandoned trials: a call for people to publish the findings. BMJ. 2013; 346:f2865. doi:10.1136/bmj.f2865.

31. Rodgers MA, Brown JV, Heirs MK, Higgins JP, Mannion RJ, Simmonds MC, et al. Reporting of industry funded study outcome data: comparison of confidential and published data on the safety and effectiveness of rhBMP-2 for spinal fusion. BMJ. 2013:346:f3981-1.

32. Maund E, Tendal B, Hróbjartsson A, Jørgensen KJ, Lundh A, Schroll J, et al. Benefits and harms in clinical trials of duloxetine for treatment of major depressive disorder: comparison of clinical study reports, trial registries, and publications. BMJ. 2014;4(348):g3510. doi:10.1136/bmj.g3510.

33. REGULATION (EU) No 536/2014 OF THE EUROPEAN PARLIAMENT AND OF THE COUNCIL of 16 April 2014. On clinical trials on medicinal products for human use, and repealing Directive 2001/20/EC. Official Journal of the
European Union. Available at: http://eur-lex.europa.eu/legal-content/EN/TXT/ ?uri=uriserv:OJ.L_.2014.158.01.0001.01.ENG. Accessed 20 Jun 2015.

34. Le Noury J, Nardo JM, Healy D, Jureidini J, Raven M, Tufanaru C, et al. Restoring Study 329: efficacy and harms of paroxetine and imipramine in treatment of major depression in adolescence. BMJ. 2015;351:h4320. doi:10.1136/bmj.h4320.

\section{Submit your next manuscript to BioMed Central and we will help you at every step:}

- We accept pre-submission inquiries

- Our selector tool helps you to find the most relevant journal

- We provide round the clock customer support

- Convenient online submission

- Thorough peer review

- Inclusion in PubMed and all major indexing services

- Maximum visibility for your research

Submit your manuscript at www.biomedcentral.com/submit
C Biomed Central 